\title{
Why are Temperature and Upward Wave Activity Flux Positively Skewed in the Polar Stratosphere?
}

\author{
OLIVER WATT-MEYER \\ Department of Atmospheric Sciences, University of Washington, Seattle, Washington \\ PAUL J. KUSHNER \\ Department of Physics, University of Toronto, Toronto, Ontario, Canada
}

(Manuscript received 7 March 2017, in final form 25 July 2017)

\begin{abstract}
The distribution of temperatures in the wintertime polar stratosphere is significantly positively skewed, which has important implications for the characteristics of ozone chemistry and stratosphere-troposphere coupling. The typical argument for why the temperature distribution is skewed is that radiative balance sets a firm lower limit, while planetary wave driving can force much larger positive anomalies in temperature. However, the distribution of the upward Eliassen-Palm (EP) flux is also positively skewed, and this suggests that dynamics may play an important role in setting the skewness of the temperature distribution. This study explains the skewness of the upward EP flux distribution by appealing to the ideas of linear interference. In this framework, fluxes are decomposed into a linear term (LIN) that measures the coherence of the wave anomaly and the climatological wave and an additional nonlinear term (NONLIN) that depends only on the wave anomaly. It is shown that when filtered by wavenumber, there is a clear nonlinear dependence between LIN and NONLIN: the terms cancel when LIN is negative, but they reinforce each other when LIN is positive. This leads to the positive skewness of the upward wave activity flux. A toy model of wave interference is constructed, and it is shown that the westward vertical tilt of the climatological wave is the key ingredient to a positively skewed upward EP flux distribution. The causes of the skews of the LIN and NONLIN distributions themselves are shown to be related to relationships between wave phase and amplitude, and wave phase and vertical tilt, respectively.
\end{abstract}

\section{Introduction}

Since early work documenting the variability of the Northern Hemisphere stratospheric polar vortex, it has been known that the distribution of temperatures in the wintertime vortex is positively skewed (Labitzke 1982; Gillett et al. 2001; Yoden et al. 2002). ${ }^{1}$ Because temperature in the polar stratospheric vortex is well correlated with the northern annular mode (NAM) (Thompson and Wallace 2000), it is not surprising that there is also a skew in the distribution of the NAM

\footnotetext{
${ }^{1}$ Throughout this study, the skewness is calculated as the scaled third moment of the distribution, that is, using the formula $s=E\left[(x-\mu)^{3}\right] / \sigma^{3}$, where $\mu$ is the mean and $\sigma$ is the standard deviation of the given distribution $x$ (e.g., section 2.6.7 of von Storch and Zwiers 1999).
}

Corresponding author: Oliver Watt-Meyer, oliverwm@uw.edu in the stratosphere (Gillett et al. 2001). These skews are found both in monthly mean data (Labitzke 1982; Yoden et al. 2002) and daily data (Gillett et al. 2001). Understanding the distribution of winds and temperatures in the stratosphere is important because the lower (cold) end of the distribution is fundamental for ozone chemistry, and in particular photochemical ozone loss, in the stratosphere. Some winters with extended anomalously cold temperatures, like 2010/ 11 , have led to substantial amounts of ozone loss in the Northern Hemisphere (e.g., Manney et al. 2011). Furthermore, extreme warm conditions in the polar stratosphere are associated with weak polar vortex events, which tend to be followed by a persistent negative NAM signature in the troposphere (Baldwin and Dunkerton 2001). Because of this, knowledge of the conditions determining stratospheric circulation extremes can improve predictions of tropospheric weather. 
The typical explanation for a positively skewed distribution in winter temperatures is that dynamical wave driving can force relatively large positive anomalies in temperature (and coincident weakening in the stratospheric circulation) while there is a firm lower bound on temperatures set by radiative balance (Gillett et al. 2001). However, recent work has shown that wave driving can also force vortex accelerations and negative temperature anomalies in the polar stratosphere (Shaw and Perlwitz 2013, 2014; Dunn-Sigouin and Shaw 2015). In addition, as will be shown in this study, the upward wave activity flux distribution is itself positively skewed in the lower stratosphere. Given the strong connection between the upward wave activity flux in the lower stratosphere and polar vortex temperature and strength (Newman et al. 2001; Polvani and Waugh 2004), these two facts suggest that the positive skewness of the temperature distribution may be more dynamically controlled than typically thought.

Although our ultimate motivation is to understand the distribution of temperatures in the stratosphere, this study will focus on explaining the positive skewness of the upward wave activity flux distribution, which is known to be closely connected to the temperatures in the stratosphere (Newman et al. 2001; Polvani and Waugh 2004). We will begin by outlining the observed distributions of temperature and upward wave activity flux in the stratosphere and show that both are positively skewed. The skewness of the heat flux distribution will be explained by appealing to the ideas of linear interference (Nishii et al. 2009; Garfinkel et al. 2010; Smith and Kushner 2012). Linear interference is a useful framework for understanding the variability of flux quantities in the presence of climatological zonal asymmetries. It separates quantities such as the meridional heat flux into two terms, one that represents the interference between the wave anomaly and climatological wave, and the other that is the heat flux solely resulting from the wave anomaly itself. We will show a novel result that, when examined at each individual wavenumber, there is a clear nonlinear relationship between each of these terms. An argument based on wave anomaly tilts is proposed to explain this relationship, and it is used to provide an explanation for the positive skewness of the upward wave activity flux. Finally, a simple toy model of wave interference is developed in order to explore the key parameters that set the positive skewness of the wave activity flux distribution. This model uses artificially generated distributions of the wave anomaly amplitude, phase, and vertical tilt, as well as prescribed values for the climatological wave amplitude, phase, and tilt. It is shown that highly simplified distributions of the wave anomaly parameters can be used, but that for the wave-1 Northern Hemisphere heat flux distribution, the westward tilt with height of the climatological wave is essential in obtaining a positively skewed heat flux distribution. While this study focuses on the Northern Hemisphere's largest horizontal scale, planetary wave 1 , the distributions for the wave- 2 heat flux and the Southern Hemisphere will be discussed briefly.

\section{Data and methods}

The 1979-2013 daily-mean geopotential height, meridional wind, and temperature data on a $1.5^{\circ} \times 1.5^{\circ}$ grid from ERA-Interim are used (Dee et al. 2011). Data from the NCEP-NCAR Reanalysis-1 (Kalnay et al. 1996) over the years 1958-2011 have also been used, with no qualitative difference in the results (not shown). The daily climatology (computed as a simple average over all years for that calendar day) of a variable $a$ is denoted as $a_{c}$, and the anomaly from the climatology is written as $a^{\prime}$; that is, $a=a_{c}+a^{\prime}$. The zonal mean of $a$ is written as $\{a\}$, and the deviation therefrom is denoted as $a^{*}$. The focus will be on the heat flux at $100 \mathrm{hPa}$ and $60^{\circ} \mathrm{N}$. Although the heat flux averaged over some range of the mid- to high latitudes is typically used when measuring the input of wave activity flux into the stratosphere (e.g., Newman et al. 2001; Polvani and Waugh 2004), we will focus on the heat flux at a single latitude because we will ultimately be aiming to explain the heat flux distribution by appealing to simple properties of the wave anomaly, such as its amplitude, phase, and tilt. These properties are defined at a single latitude and thus are most straightforward to compare with the heat flux at a single latitude. Regardless, the correlation between $100-\mathrm{hPa}\left\{v^{*} T^{*}\right\}^{\prime}$ at $60^{\circ} \mathrm{N}$ and that averaged between $45^{\circ}$ and $75^{\circ} \mathrm{N}$, computed over all DecemberFebruary (DJF) days, is 0.97 for wave 1 and 0.98 for wave 2 , and so we would not expect a qualitative change in the results if we were to use the meridionally averaged heat flux. We will primarily examine the zonal wave- 1 component of the heat flux, since this is the dominant term in the lower stratosphere, but a comparison with wave 2 will be discussed in section $3 \mathrm{f}$. The focus will be on seasons with the largest variability of the upward wave activity flux, that is, DJF in the Northern Hemisphere (Fig. 2 of Smith and Kushner 2012) and September-November (SON) in the Southern Hemisphere (Fig. 9 of Smith and Kushner 2012).

\section{a. Linear interference}

In the presence of strong zonal asymmetries in the climatological state of the atmosphere, heat flux anomalies tend to be at their extremes when the wave anomaly 
is either in or out of phase with the climatological wave (e.g., Fig. 1 of Watt-Meyer and Kushner 2015b). Furthermore, changes in the strength of the stratospheric polar vortex tend to be preceded by wave anomalies that either amplify or attenuate the climatology (Garfinkel et al. 2010; Smith and Kushner 2012; Watt-Meyer and Kushner 2015b). This effect can be quantified as follows. By separating the meridional wind and temperature zonal eddies into climatological and anomaly components- $v^{*}=v_{c}^{*}+v^{* \prime}$ and $T^{*}=T_{c}^{*}+T^{* \prime}$, respectively - the anomalous meridional heat flux can be written as (e.g., Smith and Kushner 2012)

$$
\begin{aligned}
\left\{v^{*} T^{*}\right\}^{\prime} & =\left\{v^{* \prime} T_{c}^{*}\right\}+\left\{v_{c}^{*} T^{* \prime}\right\}+\left\{v^{*^{\prime}} T^{* \prime}\right\}^{\prime} \\
& =\text { LIN + NONLIN },
\end{aligned}
$$

where

$$
\begin{aligned}
\text { LIN } & =\left\{v^{* \prime} T_{c}^{*}\right\}+\left\{v_{c}^{*} T^{* \prime}\right\} \quad \text { and } \\
\text { NONLIN } & =\left\{v^{*^{\prime}} T^{*^{\prime}}\right\}-\left\{v^{*^{\prime}} T^{*^{\prime}}\right\}_{c}=\left\{v^{*^{\prime}} T^{* \prime}\right\}^{\prime} .
\end{aligned}
$$

In the next section, analytic expressions will be derived for the linear (LIN) and nonlinear (NONLIN) terms for a single-wavenumber disturbance as a function of geopotential height anomaly wave amplitude, phase, and tilt, assuming geostrophic and hydrostatic balance. Briefly, the LIN term depends linearly on the anomalous and climatological wave amplitudes, depends linearly on the sum of the anomaly and climatological wave tilts, and goes as the cosine of the difference of the wave anomaly and climatological wave phase. The NONLIN term has a quadratic dependence on the wave anomaly amplitude and depends linearly on the wave anomaly tilt: it is larger for westward-tilted anomalies and smaller for eastward-tilted anomalies.

\section{b. Toy model of wave interference}

In section $3 \mathrm{~d}$ a simple model of wave interference will be used to examine the importance of various aspects of the climatological wave and of the wave anomaly distribution for the positive skewness of the heat flux distribution. Here, we derive the necessary analytic expressions for use in that model. We consider the geopotential height anomaly and climatology and use hydrostatic and geostrophic balances to compute the corresponding temperature and meridional wind, and thus the heat flux. Disturbances of a single-wavenumber mode will be considered. Because we are interested in the heat flux at one particular latitude and pressure level, we just need the amplitude, phase, and tilt of the wave anomaly and of the climatological wave at that level (the tilt is necessary in order to compute the temperature from the geopotential height). The observed distributions of these three parameters for wave 1 in the high-latitude lower stratosphere will be shown in section 3c. To derive expressions for the total heat flux, as well as the LIN and NONLIN terms, as a function of the wave amplitude, phase, and tilt, we begin by assuming the geopotential height at some particular latitude and time takes the form

$$
Z^{*}(\lambda, p)=A(p) \cos [k \lambda-\theta(p)],
$$

where $A(p)$ is the pressure-dependent wave amplitude, $k$ is the zonal wavenumber, and $\theta(p)$ is the pressuredependent wave phase. Given the typical scaling of the wave amplitude as the inverse square root of pressure [e.g., Eq. (4.2.3a) of Andrews et al. 1987], we will assume that

$$
A(z)=A_{0} e^{z / 2 H},
$$

with $A_{0}=A\left(z_{0}\right), z=H \ln \left(p_{0} / p\right)$, and $H$ is a density scale height. This can be shown to imply

$$
A(p)=A\left(p_{0}\right) \sqrt{\frac{p_{0}}{p}}
$$

and

$$
\frac{d A}{d \ln p}=-H \frac{d A}{d z}=-\frac{A}{2} .
$$

How well Eq. (6) holds for wave 1 in the extratropical lower stratosphere is tested by computing the correlation between the actual amplitudes at $60^{\circ} \mathrm{N}$ for levels adjacent to $100 \mathrm{hPa}$ (i.e., 125 and $70 \mathrm{hPa}$ in ERAInterim) against estimates of the amplitude at these adjacent levels computed using the true amplitude at $100 \mathrm{hPa}$ and Eq. (6). These correlations are $r=0.97$ and $r=0.95$ for 125 and $70 \mathrm{hPa}$, respectively. Furthermore, scatterplots between the estimated and true amplitudes fall closely on the one-to-one line (not shown). This indicates the appropriateness of the assumption of wave amplitude being inversely proportional to the square root of pressure. Equation (7) will be used below when deriving an expression for the temperature in terms of the phase, amplitude, and tilt of the geopotential height wave at some particular pressure level.

Next, hydrostatic balance [e.g., Eq. (1.1.4) of Andrews et al. 1987] is used to compute the temperature from Eq. (4):

$$
\begin{aligned}
T^{*}(\lambda, p)= & -\frac{g}{R} \frac{\partial Z^{*}}{\partial \ln p} \\
= & -\frac{g}{R} \frac{d A(p)}{d \ln p} \cos [k \lambda-\theta(p)] \\
& -\frac{g}{R} A(p) \frac{d \theta(p)}{d \ln p} \sin [k \lambda-\theta(p)],
\end{aligned}
$$


where $g$ is the acceleration due to gravity, and $R$ is the gas constant for dry air. Substituting in Eq. (7), we have

$$
\begin{aligned}
& T^{*}(\lambda, p) \\
& \quad=\frac{g}{R} A(p)\left\{\frac{1}{2} \cos [k \lambda-\theta(p)]-\frac{d \theta(p)}{d \ln p} \sin [k \lambda-\theta(p)]\right\} .
\end{aligned}
$$

In Eq. (9), $d \theta(p) / d \ln p$ is the tilt of the wave. It is positive for a westward-tilting wave (i.e., a wave that tilts westward with increasing height or decreasing pressure) and negative for an eastward-tilting wave. Now applying the assumption of geostrophic balance to compute the meridional wind [e.g., Eq. (5.2.4) of Andrews et al. 1987], we find

$$
\begin{aligned}
v^{*}(\lambda, p) & =\frac{g}{f} \frac{1}{a \cos (\phi)} \frac{\partial Z^{*}}{\partial \lambda} \\
& =-\frac{k g}{f} \frac{1}{a \cos (\phi)} A(p) \sin [k \lambda-\theta(p)],
\end{aligned}
$$

where $a$ is the radius of Earth, $\phi$ is the latitude, and $f$ is the Coriolis frequency. Using Eqs. (9) and (10), the total heat flux for a single-wavenumber perturbation is

$$
\begin{aligned}
\left\{v^{*} T^{*}\right\} & =\frac{1}{2 \pi} \int_{0}^{2 \pi} v^{*}(\lambda, p) T^{*}(\lambda, p) d \lambda \\
& =\frac{k g^{2} A^{2}}{2 \pi f R a \cos (\phi)}\left[-\frac{1}{2} \int_{0}^{2 \pi} \cos (k \lambda-\theta) \sin (k \lambda-\theta) d \lambda+\frac{d \theta}{d \ln p} \int_{0}^{2 \pi} \sin ^{2}(k \lambda-\theta) d \lambda\right] \\
& =\frac{k g^{2} A^{2}}{2 f R a \cos (\phi)} \frac{d \theta}{d \ln p}
\end{aligned}
$$

The NONLIN term [see Eq. (3)] can be calculated from Eq. (11) by using the parameters for the wave anomaly instead of the total wavefield; that is,

$$
\text { NONLIN }=\frac{k g^{2}\left(A^{\prime}\right)^{2}}{2 f R a \cos (\phi)} \frac{d \theta^{\prime}}{d \ln p}-\left[\frac{k g^{2}\left(A^{\prime}\right)^{2}}{2 f R a \cos (\phi)} \frac{d \theta^{\prime}}{d \ln p}\right]_{c},
$$

where $A^{\prime}$ and $d \theta^{\prime} / d \ln p$ are the amplitude and tilt of the anomalous wave, respectively. The second term in Eq. (12), which depends only on the day of year and is positive throughout the Northern Hemisphere winter, guarantees that NONLIN has zero climatological mean [cf. Eq. (3)]. Equation (12) shows that the NONLIN term has a quadratic dependence on the amplitude of the wave anomaly and a linear dependence on the phase tilt. In particular, it will be greater for a westward-tilting wave and smaller for an eastward-tilting wave.

For the LIN term we must consider the fact that the wave phases, tilts, and amplitudes will be different for the climatology versus the anomaly. Below, an expression for the LIN term is derived as a function of the anomaly amplitude, phase, and tilt, and climatology amplitude, phase, and tilt. With the anomaly and climatology geopotential heights given by

$$
\begin{aligned}
Z^{*}(\lambda, p) & =A^{\prime}(p) \cos \left[k \lambda-\theta^{\prime}(p)\right] \quad \text { and } \\
Z_{c}^{*}(\lambda, p) & =A_{c}(p) \cos \left[k \lambda-\theta_{c}(p)\right],
\end{aligned}
$$

and making the same assumptions for the amplitude height dependence and geostrophic and hydrostatic balance as above, it can be shown that

$$
\begin{aligned}
\left\{v_{c}^{*} T^{* \prime}\right\} & =\frac{1}{2 \pi} \int_{0}^{2 \pi} v_{c}^{*} T^{*^{\prime}} d \lambda \\
& =\frac{g^{2} k A_{c} A^{\prime}}{2 \pi f a R \cos (\phi)}\left[-\frac{1}{2} \int_{0}^{2 \pi} \sin \left(k \lambda-\theta_{c}\right) \cos \left(k \lambda-\theta^{\prime}\right) d \lambda+\frac{d \theta^{\prime}}{d \ln p} \int_{0}^{2 \pi} \sin \left(k \lambda-\theta_{c}\right) \sin \left(k \lambda-\theta^{\prime}\right) d \lambda\right] \\
& =\frac{g^{2} k A_{c} A^{\prime}}{2 f a R \cos (\phi)}\left[-\frac{1}{2} \sin \left(\theta^{\prime}-\theta_{c}\right)+\frac{d \theta^{\prime}}{d \ln p} \cos \left(\theta^{\prime}-\theta_{c}\right)\right] .
\end{aligned}
$$

And similarly, 


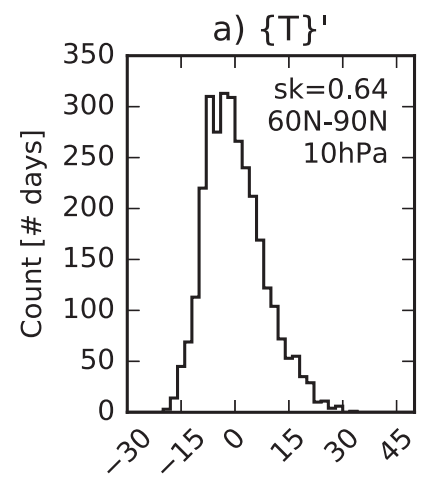

Temperature $[\mathrm{K}]$

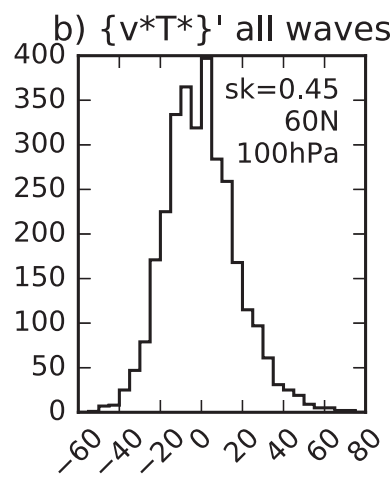

Heat Flux $[\mathrm{K} \mathrm{m} / \mathrm{s}]$

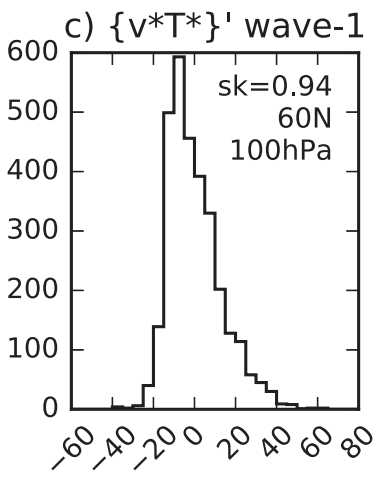

Heat Flux $[\mathrm{K} \mathrm{m} / \mathrm{s}]$

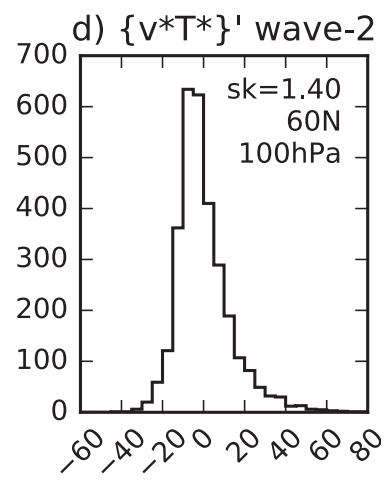

Heat Flux $[\mathrm{K} \mathrm{m} / \mathrm{s}]$

FIG. 1. Daily histograms over all DJF days of (a) $\{T\}^{\prime}$ at $10 \mathrm{hPa}$ and averaged from $60^{\circ}$ to $90^{\circ} \mathrm{N}$ with a cosine of latitude weighting, (b) $\left\{v^{*} T^{*}\right\}^{\prime}$, (c) wave-1 $\left\{v^{*} T^{*}\right\}^{\prime}$, and (d) wave-2 $\left\{v^{*} T^{*}\right\}^{\prime}$-all at $100 \mathrm{hPa}$ and $60^{\circ} \mathrm{N}$. The skew of each distribution is shown in the top-right corner of each panel.

$$
\begin{aligned}
\left\{v^{* \prime} T_{c}^{*}\right\} & =\frac{1}{2 \pi} \int_{0}^{2 \pi} v^{*^{\prime}} T_{c}^{*} d \lambda \\
& =\frac{g^{2} k A_{c} A^{\prime}}{2 \pi f a R \cos (\phi)}\left[-\frac{1}{2} \int_{0}^{2 \pi} \sin \left(k \lambda-\theta^{\prime}\right) \cos \left(k \lambda-\theta_{c}\right) d \lambda+\frac{d \theta_{c}}{d \ln p} \int_{0}^{2 \pi} \sin \left(k \lambda-\theta^{\prime}\right) \sin \left(k \lambda-\theta_{c}\right) d \lambda\right] \\
& =\frac{g^{2} k A_{c} A^{\prime}}{2 f a R \cos (\phi)}\left[\frac{1}{2} \sin \left(\theta^{\prime}-\theta_{c}\right)+\frac{d \theta_{c}}{d \ln p} \cos \left(\theta^{\prime}-\theta_{c}\right)\right] .
\end{aligned}
$$

Thus,

$$
\begin{aligned}
\mathrm{LIN} & =\left\{v_{c}^{*} T^{*^{\prime}}\right\}+\left\{v^{* \prime} T_{c}^{*}\right\} \\
& =\frac{g^{2} k A^{\prime} A_{c}}{2 f a R \cos (\phi)}\left(\frac{d \theta^{\prime}}{d \ln p}+\frac{d \theta_{c}}{d \ln p}\right) \cos \left(\theta^{\prime}-\theta_{c}\right) .
\end{aligned}
$$

Equation (16) demonstrates the dependence of the LIN term on the difference between the phase of the wave anomaly and the climatological wave. Assuming that both the anomalous and climatological waves have a westward tilt with height-that is, $d \theta^{\prime} / d \ln p>0$ and $d \theta_{c} / d \ln p>0$ - then the sign of LIN is determined by the relative phases of the climatological and anomalous waves. It will be positive if $\left|\theta^{\prime}-\theta_{c}\right|<\pi / 2$ and negative otherwise. Furthermore, Eq. (16) shows that the LIN term has a linear dependence on the anomaly amplitude and that it depends on the sum of the tilts of the climatological and anomalous waves. If the anomalous wave has an eastward tilt with height that is of comparable magnitude to the climatological wave's westward tilt, then the LIN term will be close to zero. Because of this, if the climatological wave has a westward tilt with height (as is the case for wave 1 and wave 2 in the Northern Hemisphere), then a strongly positive or negative LIN is an indication of a westward-tilted anomalous wave.

The dependencies of the LIN and NONLIN terms on the wave amplitudes, tilts, and phases have been noted qualitatively by previous authors (e.g., Smith and Kushner 2012) but until now they have not been explicitly calculated analytically.

\section{Results}

\section{a. Temperature and heat flux distributions}

Zonal-mean polar stratospheric temperatures in the Northern Hemisphere winter are known to be positively skewed (Labitzke 1982; Gillett et al. 2001; Yoden et al. 2002). This section documents the temperature distributions and additionally shows that the lowerstratospheric heat flux distribution is also positively skewed. Figure 1 shows the daily histograms of DJF polar cap temperature in the midstratosphere and highlatitude heat flux in the lower stratosphere. The polar cap stratospheric temperature anomalies have a range from approximately -20 to $30 \mathrm{~K}$ and have a skew of 0.64 (Fig. 1a). The heat flux anomaly at $100 \mathrm{hPa}$ and $60^{\circ} \mathrm{N}$ is also positively skewed, with values of $0.45,0.94$, and 1.40 for the total, wave-1, and wave- 2 components, respectively. These skews and those for the Southern Hemisphere, which will be discussed in section 3f, are summarized in Table 1 . Section $3 \mathrm{~b}$ will propose an explanation for the positive skewness of the wave- 1 and wave- 2 heat fluxes based on a newly discovered relationship 
TABLE 1. Summary of skewness of heat flux distributions for observations. NH corresponds to $60^{\circ} \mathrm{N}$ and $100 \mathrm{hPa}$ during DJF, and SH corresponds to $60^{\circ} \mathrm{S}$ and $100 \mathrm{hPa}$ during SON (SH heat fluxes are multiplied by -1). Uncertainties are given as $95 \%$ confidence intervals and are computed by bootstrapping: the heat flux distributions are resampled with replacement 10000 times, and the uncertainty provided is 1.96 times the standard deviation across this distribution of skews.

\begin{tabular}{ccccc}
\hline \hline Hemisphere & Wavenumber & $\left\{v^{*} T^{*}\right\}^{\prime}$ skew & LIN skew & NONLIN skew \\
\hline NH & 1 & $0.94 \pm 0.11$ & $-0.30 \pm 0.10$ & $1.76 \pm 0.46$ \\
NH & 2 & $1.40 \pm 0.22$ & $-0.01 \pm 0.14$ & $1.61 \pm 0.27$ \\
SH & 1 & $0.79 \pm 0.11$ & $-0.20 \pm 0.16$ & $0.84 \pm 0.22$ \\
SH & 2 & $0.75 \pm 0.16$ & $-0.20 \pm 0.19$ & $0.93 \pm 0.18$ \\
\hline
\end{tabular}

between the LIN and NONLIN terms. Before discussing this relationship, the individual distributions of these components of the heat flux are shown for wave 1 (Fig. 2). As discussed in Smith and Kushner (2012), the LIN heat flux is weakly negatively skewed (Fig. 2b), while the NONLIN heat flux is positively skewed (Fig. 2c). This is in accordance with the fact that positive and negative heat flux anomaly events tend to be driven more by the NONLIN and LIN terms, respectively (Fig. 6 of WattMeyer and Kushner 2015b).

\section{b. LIN and NONLIN relationship}

Previous work has suggested a weak negative covariance between the LIN and NONLIN terms in the Northern Hemisphere winter (e.g., see Fig. 2 of Smith and Kushner 2012; Fig. 2 of Watt-Meyer and Kushner 2015b). However, these results were based on the total-that is, all wavenumbers-LIN and NONLIN fluxes. Here it is shown that when examined by individual wavenumbers, there is a clear but nonlinear relationship between the LIN and NONLIN terms for wave 1 and wave 2 . An argument based on the wave anomaly and climatological wave tilts will be made to explain the relationship, and it will be used to explain the positive skewness of wave- 1 and wave- 2 heat fluxes in the Northern Hemisphere.
Figure 3 shows 2D histograms of LIN versus NONLIN and LIN versus $\left\{v^{*} T^{*}\right\}^{\prime}$, and reproduces the 1D histograms of $\left\{v^{*} T^{*}\right\}^{\prime}$ for all wavenumbers, wave 1 plus wave 2 , wave 1 , and wave 2 . Focusing first on the LIN versus NONLIN distributions, note that when including all wavenumbers (Fig. 3a) or the sum of wave 1 and wave 2 (Fig. 3d), there is no strong relationship between the variables, although the slight tendency for days to fall in the second quadrant (i.e., negative LIN and positive NONLIN) leads to a weak negative correlation of $r=-0.13$ between the two for all wavenumbers. However, when the heat fluxes are filtered for just wave 1 (Fig. 3g), a nonlinear relationship between the two terms becomes immediately apparent: when the LIN term is either strongly negative or positive, the NONLIN term tends to be positive. For wave 2 (Fig. 3j) the terms have a similar relationship, although it is slightly noisier. The dependence between these terms implies that they tend to cancel each other when LIN is negative and amplify each other when LIN is positive. This is seen explicitly in Fig. 3h: when the wave- 1 LIN is negative, the wave- $1\left\{v^{*} T^{*}\right\}^{\prime}$ stays roughly constant, whereas when the wave- 1 LIN is positive, there are large positive excursions in the $\left\{v^{*} T^{*}\right\}^{\prime}$. This leads to a positively skewed wave- 1 heat flux distribution, as the histogram in Fig. 3i shows. a) $\left\{\mathrm{V}^{*} \mathrm{~T} *\right\}$ ' wave-1

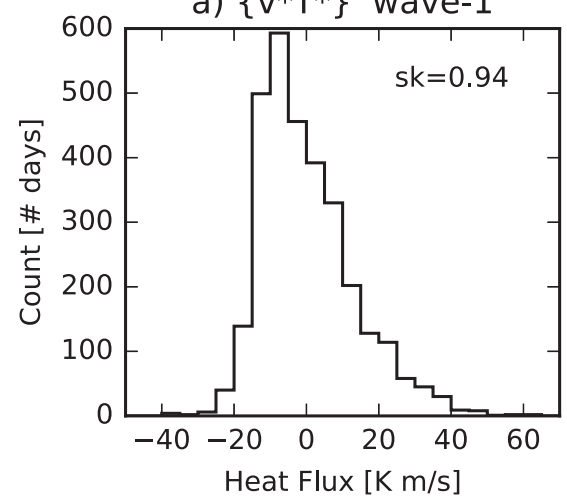

b) LIN wave-1

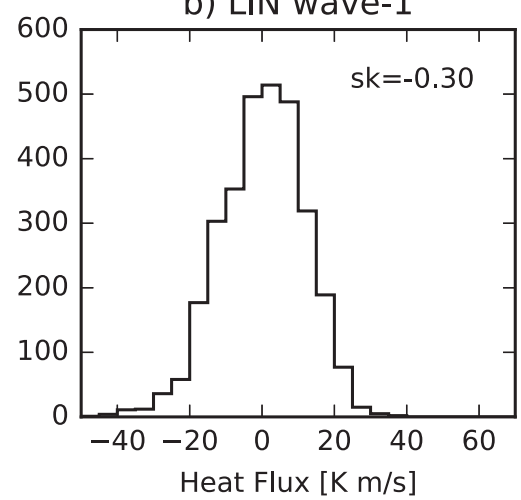

c) NONLIN wave-1

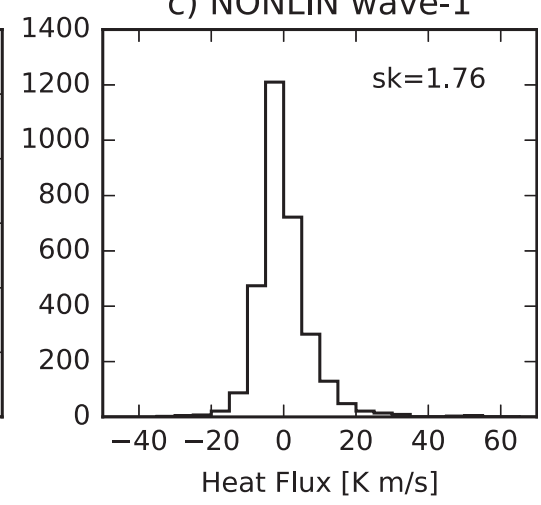

FIG. 2. Daily histograms over all DJF days of wave-1 (a) $\left\{v^{*} T^{*}\right\}^{\prime}$, (b) LIN, and (c) NONLIN at $100 \mathrm{hPa}$ and $60^{\circ} \mathrm{N}$. The skew of each distribution is written in the top-right corner of each panel, and (a) is a reproduction of Fig. 1c. 


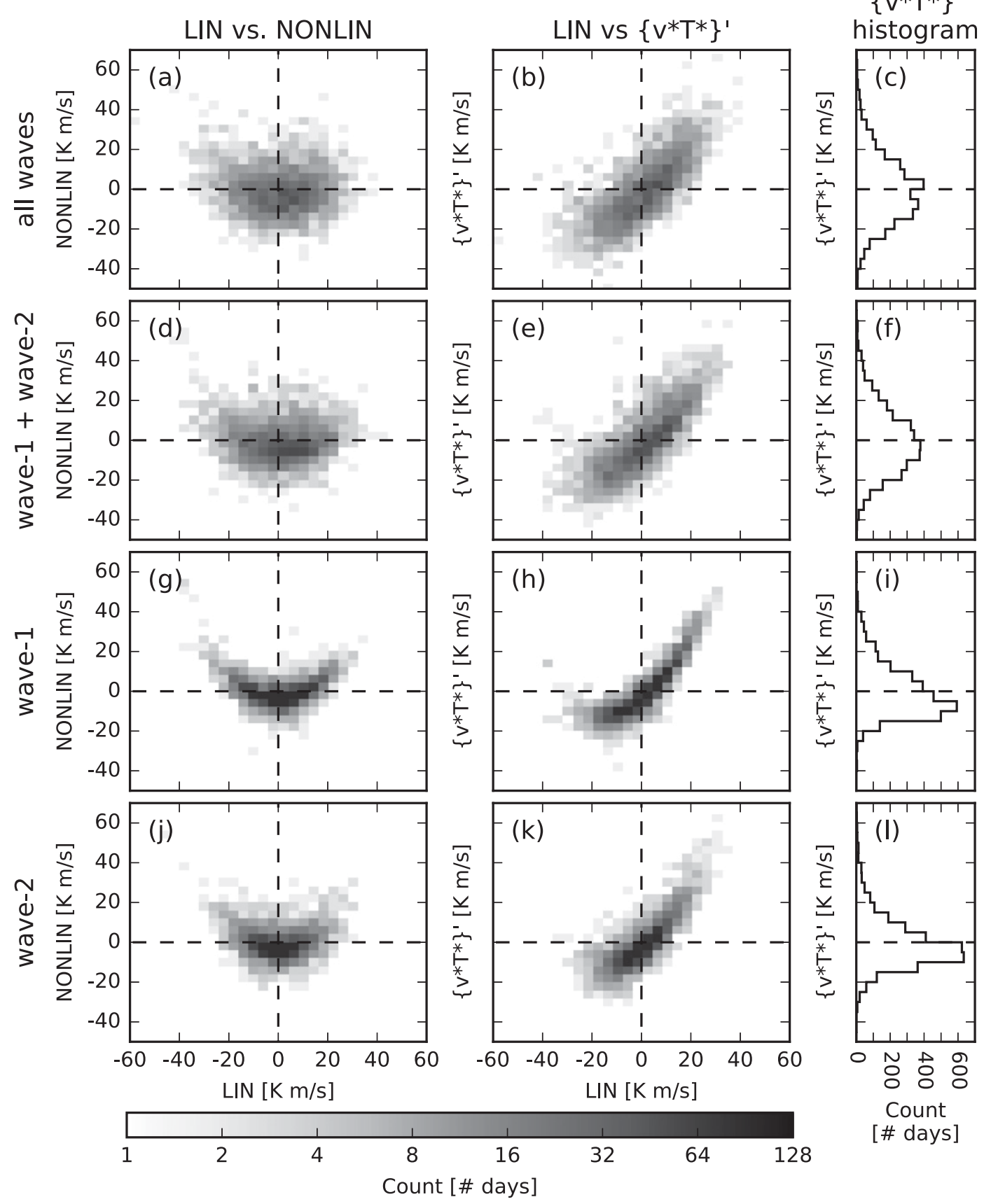

FIG. 3. Histograms of various components of the heat flux at $60^{\circ} \mathrm{N}$ and $100 \mathrm{hPa}$ over all DJF days. (left) Joint 2D histograms of LIN and NONLIN, (center) joint 2D histograms of LIN and $\left\{v^{*} T^{*}\right\}^{\prime}$, and (right) 1D histograms of $\left\{v^{*} T^{*}\right\}^{\prime}$. (a)-(c) All wavenumbers, (d)-(f) wave $1+$ wave 2, (g)-(i) wave 1, and (j)-(l) wave 2 . The grayscale for the 2D histograms is logarithmic and is the same in all plots; (c), (i), and (l) are as in Figs. 1b-d, respectively.

Furthermore, experiments with the toy model in section $3 \mathrm{~d}$ will show that the negative and positive skews of the wave-1 LIN and wave-1 NONLIN terms, respectively (recall Figs. $2 \mathrm{~b}$ and $2 \mathrm{c}$ ), are not necessary requirements for the positive skewness of the total wave- 1 heat flux anomaly.

The cause of the association between the LIN and NONLIN terms seen in Figs. $3 g$ and $3 \mathrm{j}$ can be understood 
as follows. Given that the wave- 1 and wave- 2 components of the Northern Hemisphere climatological wave (i.e., $Z_{c}^{*}$ ) at $60^{\circ} \mathrm{N}$ have a westward tilt with height throughout the troposphere and stratosphere, a strongly positive or negative LIN term implies that the wave anomaly will also have a westward tilt with height and be either in phase or out of phase with the climatological wave [recall Eq. (16) and the discussion thereafter]. However, since the NONLIN term does not depend on the phase of the wave anomaly but does depend on the tilt of the wave anomaly [Eq. (12)], for either the negative or positive LIN case, we expect a positive NONLIN term. This is precisely what is seen in Figs. $3 \mathrm{~g}$ and $3 \mathrm{j}$. The relationship between LIN and NONLIN is clearest for wave 1 , so we will primarily focus on this wavenumber and delay the discussion of wave 2 until section $3 \mathrm{f}$.

Although the relationship between the LIN and NONLIN terms is clearly evident for both wave- 1 and wave-2 fluxes (Figs. 3g and 3j), it does not exist when considering all wavenumbers (Fig. 3a). It is not immediately clear why this is, in particular given that the variance in heat flux at $60^{\circ} \mathrm{N}$ and $100 \mathrm{hPa}$ is largely driven by these planetary scales. To answer this question, first, the possibility of a higher wavenumber (specifically, wave 3 or greater) heat flux variability impacting the LIN-NONLIN relationship is eliminated. This is done by plotting the 2D histograms of LIN versus NONLIN for the sum of the wave- 1 and wave- 2 heat fluxes (Fig. 3d). This histogram is very similar to the one for the total heat fluxes and thus indicates that higher wavenumber variability is not the cause of the lack of connection between LIN and NONLIN when considering all wavenumbers. This therefore suggests that interactions between wave- 1 and wave- 2 heat fluxes are likely the important factor. Scatterplots of wave- 1 versus wave- 2 heat fluxes (not shown) demonstrate that the two wavenumbers are not entirely independent. For the total heat flux anomaly and the NONLIN term, the relationship is nonlinear, and there is a tendency for large amplitude positive events to occur independently for wave 1 and wave 2. For the LIN term, there is a negative linear relationship between the two $(r=-0.23)$. To test the importance of these interactions for the LIN-NONLIN relationship of the sum of wave 1 and wave 2 , the order of observations is randomized separately for the wave- 1 and wave- 2 heat fluxes in order to remove any dependence between wavenumbers, but maintain the dependence between LIN and NONLIN for each wavenumber. It is confirmed that that the LIN-NONLIN relationship, although weaker compared to the individual wavenumbers, is present for the sum of wave 1 and wave 2 when there is no dependence between the two wavenumbers (not shown).

\section{c. Observed wave anomaly parameter distributions}

In the next section, the toy model of wave interference based on the distributions of three parameters of the wave anomaly (amplitude $A^{\prime}$, phase $\theta^{\prime}$, and vertical tilt $d \theta^{\prime} / d \ln p$ ) will be used to explain the distributions of the different components of the heat flux and the relationship between the LIN and NONLIN terms. Before outlining the model results, the observed distributions of the three wave anomaly parameters are shown, as well as the relationships between the different parameters. It will be argued that some of the dependencies between wave anomaly parameters are responsible for the skews of the LIN and NONLIN distributions (Figs. $2 b$ and $2 \mathrm{c})$, a fact that will be confirmed with the toy model in the next section.

Figure 4 shows the distributions of the observed wave-1 geopotential height anomaly amplitude, phase, and tilt at $60^{\circ} \mathrm{N}$ and $100 \mathrm{hPa}$ over all DJF days, as well as the joint distributions between these parameters. Note that for the tilt parameter, the quantity plotted here and in subsequent figures is simply the difference between phases at the levels above and below $100 \mathrm{hPa}$, that is, $\theta(125 \mathrm{hPa})-\theta(70 \mathrm{hPa})$. This is used as a proxy for the actual slope in phase $d \theta / d \ln p$. When this quantity is positive (negative), it indicates a wave tilted westward (eastward) with height. Figure 4 demonstrates a number of important points. First, the wave anomaly amplitudes are not small: more than $62 \%$ of days have an anomaly amplitude greater than the amplitude of the climatological wave (Fig. 4a). Second, there are certain phases that wave- 1 anomalies have more frequently than other phases (note the two peaks separated by about $180^{\circ}$ in Fig. 4b), and in particular wave- 1 anomalies are more likely to be in or out of phase with the climatological wave rather than in quadrature with it. This is associated with the standing wave 1 at this level and latitude, which typically has antinodes near the longitudes of the maximum and minimum of the wave- 1 climatology (see Fig. 6 of Watt-Meyer and Kushner 2015a). Finally, the majority of days have a westward-tilted anomaly (i.e., $\left.d \theta^{\prime} / d \ln p>0\right)$, although usually the anomaly is not as strongly tilted as the climatological wave (Fig. 4c). This is consistent with the relatively weak baroclinic tilt of wave- 1 standing waves at $60^{\circ} \mathrm{N}$ (see Fig. 3a of WattMeyer and Kushner 2015b).

The distributions of the three parameters shown in Figs. $4 \mathrm{a}-\mathrm{c}$ are not sufficient to fully describe the distribution of wave- 1 anomalies at $60^{\circ} \mathrm{N}$ and $100 \mathrm{hPa}$. This is because the parameters are not independent of each other. Figures $4 \mathrm{~d}-\mathrm{f}$ show the observed joint distributions for the 
a) Amplitude
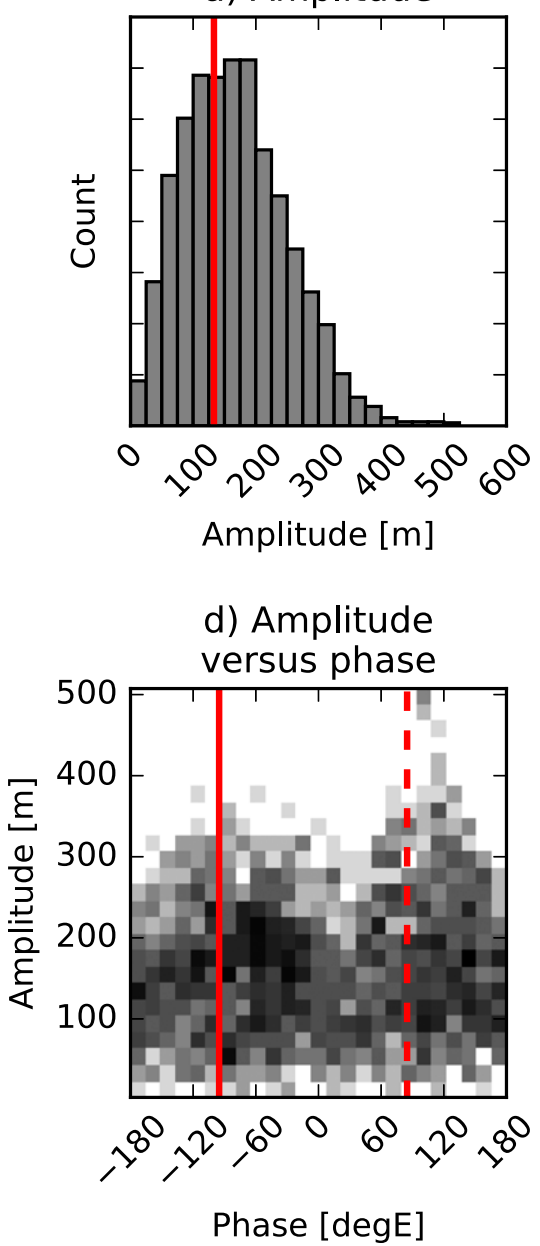

b) Phase

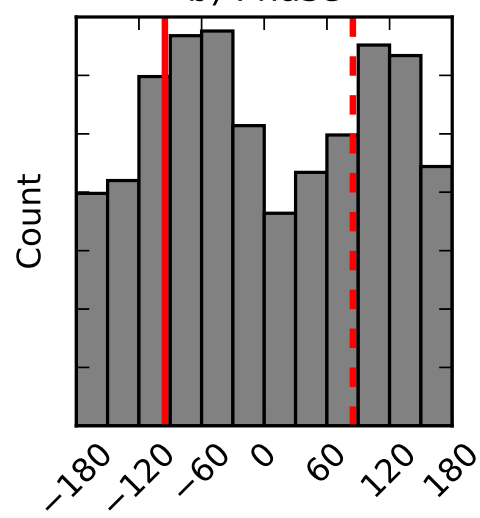

Phase [degE]

e) Amplitude versus tilt

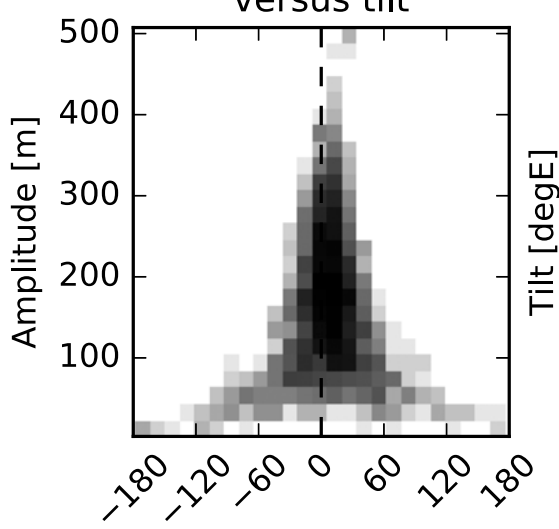

Tilt [degE]

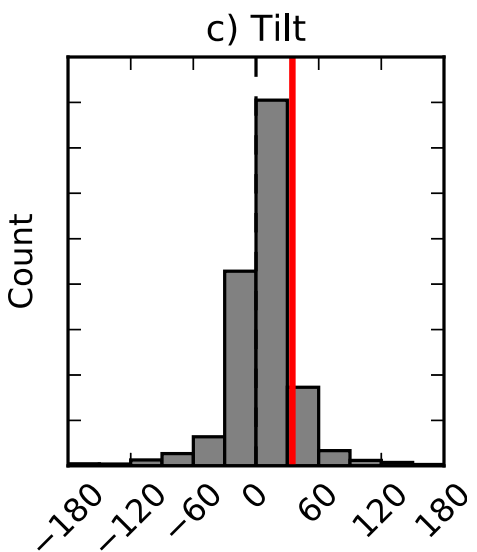

Tilt $[\operatorname{deg} E]$

f) Tilt versus phase

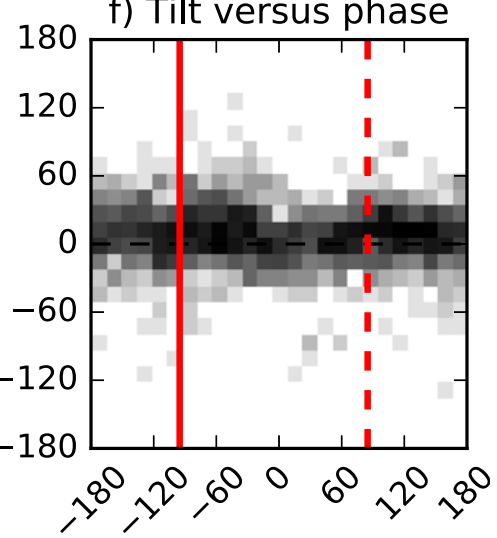

Phase [degE]

FIG. 4. Histograms of the three parameters of the geopotential height wave- 1 anomaly at $60^{\circ} \mathrm{N}$ and $100 \mathrm{hPa}$ for all DJF days: (a) the wave amplitudes $[A(p)$ in Eq. (4)], (b) the wave phase $[\theta(p)$ in Eq. (4)], and (c) the wave tilt (phase at $125 \mathrm{hPa}$ minus phase at $70 \mathrm{hPa}$ ). Twodimensional histograms of (d) amplitude vs phase, (e) amplitude vs tilt, and (f) tilt vs phase. Values of each parameter for the DJF-mean climatological wave are marked by the vertical red lines, and the phase of the minimum of the climatological wave in (b), (d), and (f) are indicated by dashed red lines.

three possible combinations of parameters. Figure $4 d$ suggests that there is a tendency for wave anomalies out of phase with the climatological wave (i.e., with phases of around $100^{\circ} \mathrm{E}$ ) to be of larger amplitude than those that are roughly in phase with the climatological wave. This explains the negative skewness of the LIN term (Fig. 2b), since wave anomalies out of phase with the climatology correspond to negative LIN. It will be confirmed in section $3 \mathrm{~d}$ that if this relationship between phase and amplitude did not exist, then the LIN distribution would be symmetric. Figure $4 \mathrm{e}$ shows that there is also a strong relationship between amplitude and tilt: the larger the anomaly amplitude, the closer to barotropic the wave anomaly tends to be. In addition to this, the largest amplitude waves are more likely to be westward tilted with height than eastward tilted: the average tilt for wave anomalies with amplitudes greater than or equal to $80 \mathrm{~m}$ is $7.73^{\circ} \mathrm{E}$, while the tilt for wave anomalies with amplitudes smaller than $80 \mathrm{~m}$ is $0.82^{\circ} \mathrm{E}$. This latter relationship is responsible for the positive skewness of the NONLIN term. This will be confirmed in section 3d by constucting a symmetric tilt distribution and showing that this leads to a symmetric NONLIN distribution. Finally, there is no clear relationship between tilt and phase (Fig. 4f).

When constructing artificial distributions of the amplitude, phase, and tilt for the toy model in the next section, simplified versions of the observed distributions will be used in order to test which of the features of the observed distributions of these parameters are required to obtain a realistic distribution of the heat flux and its components. For example, a uniform distribution in 
TABLE 2. Summary of parameter distributions used and the skewness of computed heat flux distributions for four versions of the toy model. For model 1, the observed distributions for the wave anomaly amplitude, phase, and tilt are used. For model 2, artificial distributions are generated for all three parameters. For model 3, the amplitude and tilts are chosen from the observed distributions, but the phase is chosen from a uniform distribution. For model 4, the amplitude and phase are chosen from the observed distributions, while the tilt is forced to have a symmetric distribution. See text for details. Uncertainties are given as $95 \%$ confidence intervals and are computed by bootstrapping: the heat flux distributions are resampled with replacement 10000 times, and the uncertainty provided is 1.96 times the standard deviation across this distribution of skews.

\begin{tabular}{cllllrr}
\hline \hline Model No. & Amplitude & Phase & Tilt & $\left\{v^{*} T^{*}\right\}^{\prime}$ skew & LIN skew & NONLIN skew \\
\hline 1 & Obs & Obs & Obs & $1.07 \pm 0.10$ & $-0.39 \pm 0.11$ & $1.77 \pm 0.47$ \\
2 & Lognormal & Uniform & Symm. & $1.02 \pm 0.14$ & $0.02 \pm 0.04$ & $-0.06 \pm 0.25$ \\
3 & Obs & Uniform & Obs & $1.60 \pm 0.08$ & $-0.01 \pm 0.03$ & $1.80 \pm 0.11$ \\
4 & Obs & Obs & Symm. & $0.31 \pm 0.09$ & $-0.28 \pm 0.04$ & $0.00 \pm 0.18$ \\
\hline
\end{tabular}

phase and a symmetric distribution with zero mean in tilt will be used.

\section{d. Toy model results}

As described in section $2 \mathrm{~b}$, using hydrostatic and geostrophic balances, the heat flux can be calculated using the wave anomaly amplitude, phase, and tilt (and the amplitude, phase, and tilt of the climatological wave). Four versions of the toy model will be discussed. All versions discussed in this section use the observed DJF-mean climatological parameters for $A_{c}, \theta_{c}$, and $d \theta_{c} / d \ln p$ at $60^{\circ} \mathrm{N}$ and $100 \mathrm{hPa}$, which are indicated by the red lines in Fig. 4. The first version uses the observed wave- 1 distributions of the anomaly amplitude, phase, and tilt at $60^{\circ} \mathrm{N}$ and $100 \mathrm{hPa}$ and tests whether the toy model is capable of reproducing the observed skews of the $\left\{v^{*} T^{*}\right\}^{\prime}$, LIN , and NONLIN distributions. The second version creates artificial distributions of all three parameter distributions and tests whether symmetric LIN and NONLIN distributions can still give rise to a positively skewed $\left\{v^{*} T^{*}\right\}^{\prime}$ distribution. The other two versions change only either the phase or the tilt distributions. These are constructed to demonstrate the causes of the non-Gaussianity of the LIN and NONLIN distributions. Importantly, in all cases the toy model generates a relationship between LIN and NONLIN that is qualitatively similar to the observed one (Fig. 3g) and a positively skewed $\left\{v^{*} T^{*}\right\}^{\prime}$ distribution. Table 2 summarizes the parameter distributions used for each version of the toy model, and the skews of the LIN, NONLIN, and $\left\{v^{*} T^{*}\right\}^{\prime}$ distributions for the models. Note that when using the observed parameter distributions, the model generates heat flux distributions with skews that are within the statistical uncertainty of the observed values (cf. the first rows of Tables 1 and 2).

Figure 5 shows the distributions and joint distributions for the amplitude, phase, and tilt used in toy model 2, which uses idealized distributions for all parameters. The amplitude distribution is a lognormal distribution with a location parameter $\mu=4.85$ and a scale parameter $\sigma=0.42$. The phase distribution is uniform with limits of $-180^{\circ}$ and $180^{\circ}$. The tilt distribution is constructed to have a similar relationship between amplitude and tilt as that found in observations. That is, there should be a larger spread in tilt for smaller amplitudes (see Fig. 4e). This is done as follows: once the amplitude distribution is constructed, for each value of amplitude $A$, a corresponding tilt is selected from a normal distribution with a mean of zero and a standard deviation of $200 / \sqrt{A}$. This causes the tilt to have a greater spread at lower amplitudes (see Fig. 5e). The ensemble size of each of the parameter distributions is $N=50000$.

Given the parameter distributions described above and shown in Fig. 5, and using the assumptions of hydrostatic and geostrophic balance, the heat flux, LIN, and NONLIN terms are computed using Eqs. (12) and (16). The observed relationship between wave-1 LIN and NONLIN is qualitatively reproduced by the toy model (cf. Figs. 6a and 3g). The skew of the heat flux anomaly distribution for the toy model is $1.02 \pm 0.14$ (see Fig. 6c; Table 2), similar to the observed skewness of wintertime wave- 1 heat flux of $0.94 \pm 0.11$. Importantly, toy model 2 is capable of reproducing the observed relationship between LIN and NONLIN and the positive skewness of the total heat flux anomaly, despite the fact that the parameter distributions have been simplified in comparison to the observed distributions. In particular, it is apparent that the bimodal structure of the observed phase distribution (Fig. $4 \mathrm{~b}$; indicating that wave- 1 anomalies tend to constructively or destructively interfere with the climatological wave) and the nonzero mean of the observed tilt distribution (Fig. 4c; indicating the preference for upward-propagating wave anomalies) are not necessary to recover the skewness of the total heat flux distribution.

Despite the fact that the total heat flux anomaly's positive skewness is well represented by toy model 2 , its LIN and NONLIN distributions have skews that are not significantly different from zero. This is different from 
a) Amplitude
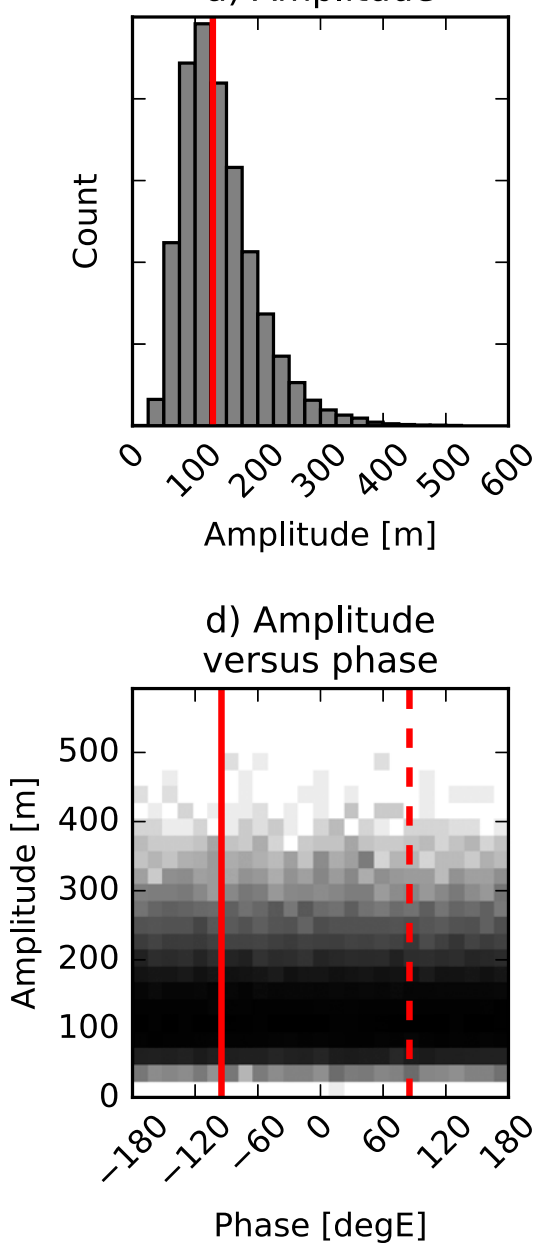

b) Phase

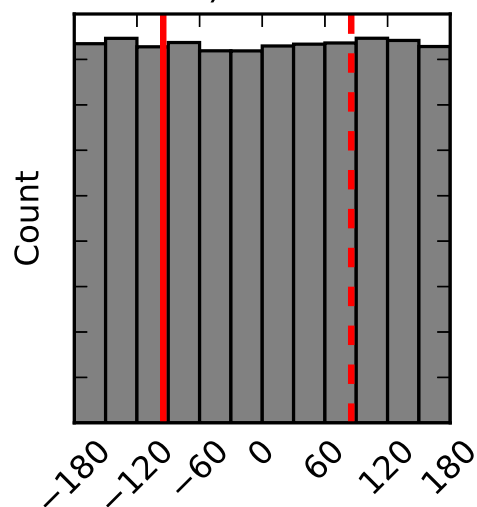

Phase [degE]

e) Amplitude versus tilt

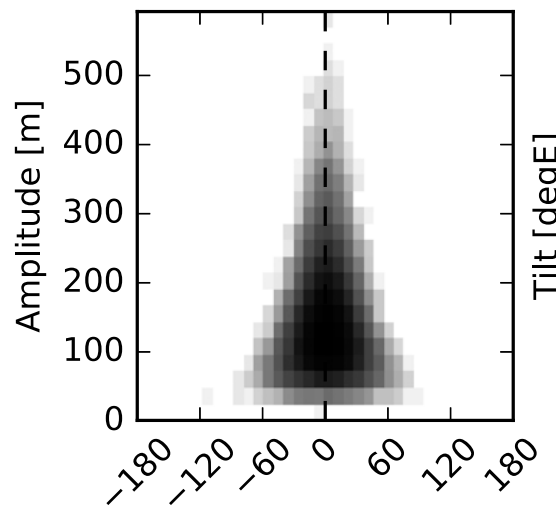

Tilt [degE] c) Tilt

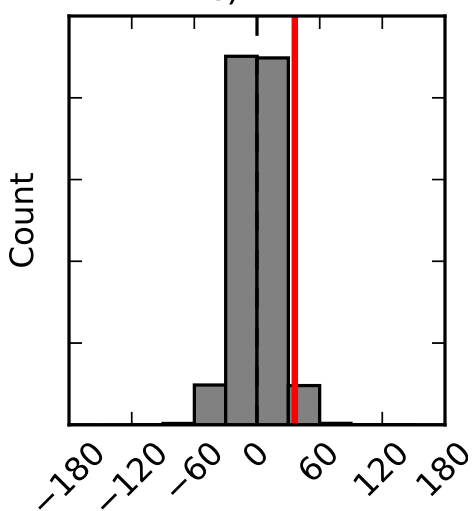

Tilt $[\operatorname{deg} E]$

f) Tilt versus phase

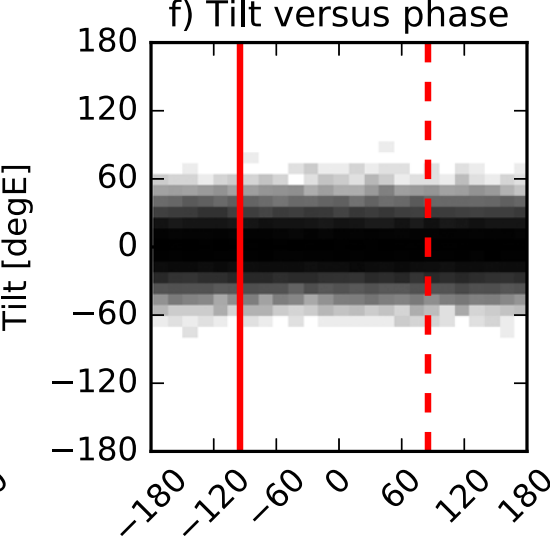

Phase [degE]

FIG. 5. As in Fig. 4, but for toy model 1.

the observed LIN and NONLIN distributions, which are negatively and positively skewed, respectively. We claim that this difference arises from the fact that toy model 2 has no dependence between amplitude and phase, and that it has a symmetric tilt distribution in which the dependence of amplitude on tilt is the same for positive and negative tilts. To show this, two additional versions of the parameter distributions are constructed (toy models 3 and 4 in Table 2). Toy model 3 samples from the observed distributions of amplitude and tilt, but it uses an independent uniform distribution for phase. It results in a LIN distribution that is not significantly different from zero. This explicitly demonstrates that the skewness of LIN is due to the observed relationship between phase and amplitude. Toy model 4 samples from the observed amplitude and phase distributions, but it forces the tilt distribution to be symmetric about zero. This is implemented as follows: for each amplitude and phase selected from the observed distributions, two sets of parameters are generated: one with the observed tilt for that day and one with the negative of the observed tilt. This preserves the main relationship between amplitude and tilt (i.e., lower magnitude of tilt for larger amplitude) but forces the tilt distribution to be symmetric. For this set of anomaly parameters (i.e., toy model 4), the NONLIN distribution has near-zero skew, confirming that the observed nonsymmetric tilt distribution leads to a positively skewed NONLIN.

\section{e. Skew dependence on climatological wave tilt}

It was claimed in the introduction that the westward tilt of the climatological wave is the essential property that leads to the positive skewness of the upward wave activity flux distribution. Here we will explore the dependence of the heat flux distribution skew on the climatological wave tilt. To begin with, its importance can be seen from Eq. (16): since $d \theta_{c} / d \ln p>0$ for a 

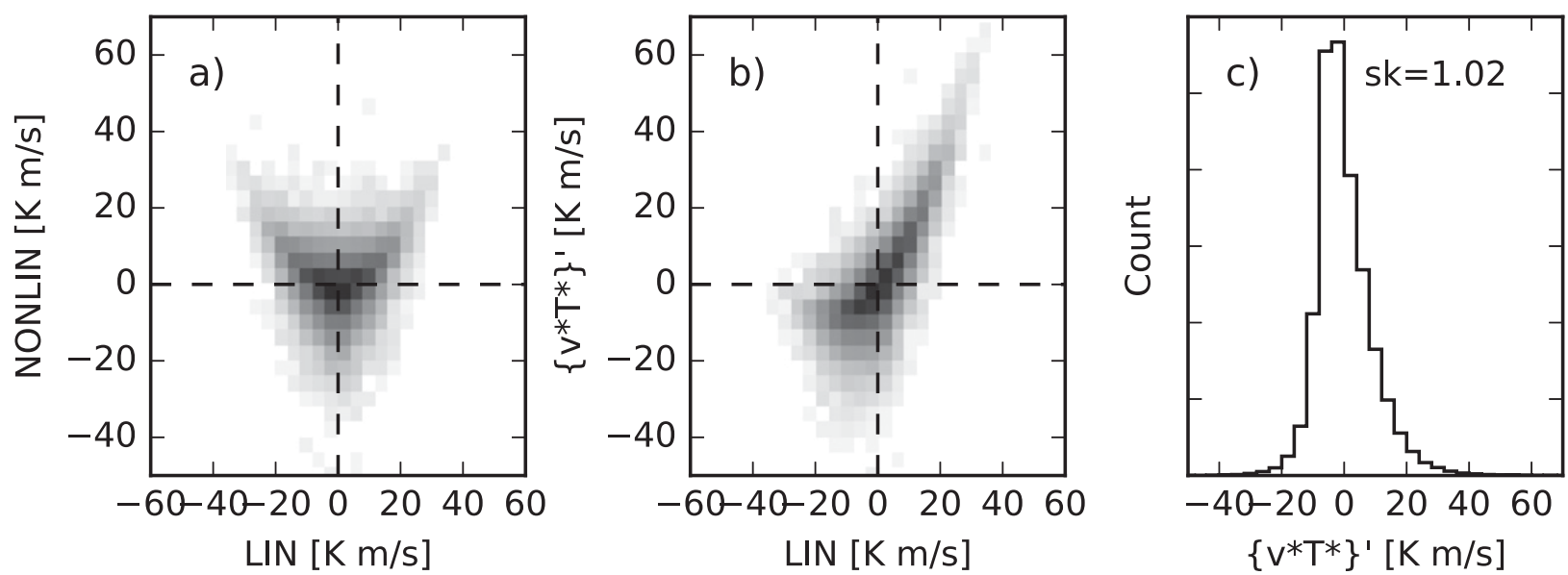

FIG. 6. For toy model 2 (see Table 2), (a) 2D histogram of LIN vs NONLIN, (b) 2D histogram of LIN vs $\left\{v^{*} T^{*}\right\}^{\prime}$, and (c) histogram of $\left\{v^{*} T^{*}\right\}^{\prime}$, as computed from the toy model. Grayscale is logarithmic in (a) and (b). Skewness of the $\left\{v^{*} T^{*}\right\}^{\prime}$ distribution is given in the upper-right corner of (c).

westward-tilted climatological wave, this means that the most extreme positive or negative LIN days tend to also have a westward-tilted anomaly $\left(d \theta^{\prime} / d \ln p>0\right)$ either in or out of phase with the climatological wave. The westward-tilted anomaly means the NONLIN term will be larger than normal [although not strictly positive because of the second term in Eq. (12)]. In turn, this means that the LIN and NONLIN terms tend to cancel when LIN is negative but amplify each other when LIN is positive, which leads to a positively skewed heat flux distribution. In this section, this idea is tested by systematically changing the tilt of the climatological wave that is prescribed for the toy model. Figure 7 shows the heat flux anomaly skew as computed by the toy model for a range of climatological wave tilts from equivalent barotropic (no tilt) to a $60^{\circ}$ difference in phase between levels above and below $100 \mathrm{hPa}$. For all the toy model runs, the anomaly parameter distributions are constructed as for toy model 2 in Table 2: that is, with a uniform phase distribution and with a symmetric tilt distribution centered at zero.

Figure 7 shows that the skew of the heat flux anomaly distribution has a strong dependence on the climatological wave tilt. In particular, it confirms that as the tilt goes to zero, the skewness also goes to zero. However, it also shows that the relationship between tilt and skew is nonmonotonic: below about $20^{\circ}$ the skew quickly increases as a function of tilt, but for greater tilts the skew slowly decreases. The observed tilt and skew are shown in Fig. 7 for both the Northern Hemisphere during DJF and the Southern Hemisphere during SON. The wave-1 climatological wave is much less tilted in the Southern Hemisphere compared to the Northern Hemisphere. The skew is also somewhat smaller for the heat flux distribution in the Southern Hemisphere. However, the two observed tilts roughly span the part of the modeled heat flux skew-tilt relationship that is approximately flat (i.e., they are on either side of the tilt that corresponds to the maximum possible heat flux skew). Thus, we cannot confidently say that the difference in heat flux skew

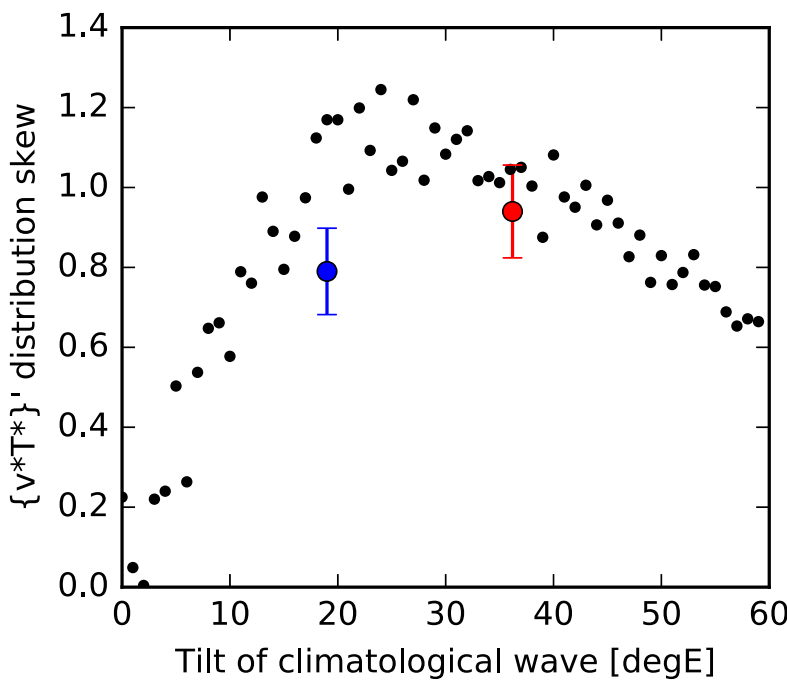

FIG. 7. Heat flux anomaly distribution's skew for the toy model as computed with varying tilts in the climatological wave (black points). For all the toy model runs, the anomaly parameter distributions are constructed as for model 2 (see Table 2), and 50000 member ensembles are created for each point. Observed heat flux skews (red point) and climatological wave tilts (blue point) in the Northern Hemisphere (at $60^{\circ} \mathrm{N}$ and $100 \mathrm{hPa}$, for DJF) and Southern Hemisphere (at $60^{\circ} \mathrm{S}$ and $100 \mathrm{hPa}$, for SON) are marked, respectively. Error bars represent $95 \%$ confidence intervals on the skew calculated using a bootstrap approach (see Table 2). SH heat flux is multiplied by -1 . 
a) $\operatorname{clim}$ tilt $=54.3^{\circ} \mathrm{E}$

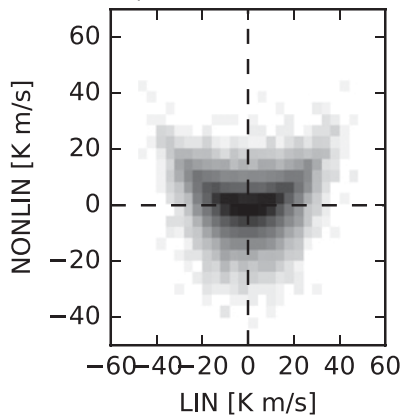

b) $\operatorname{clim}$ tilt $=36.2^{\circ} \mathrm{E}$

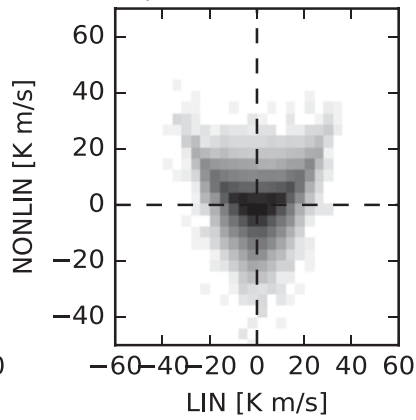

c) $\operatorname{clim}$ tilt $=18.1^{\circ} \mathrm{E}$

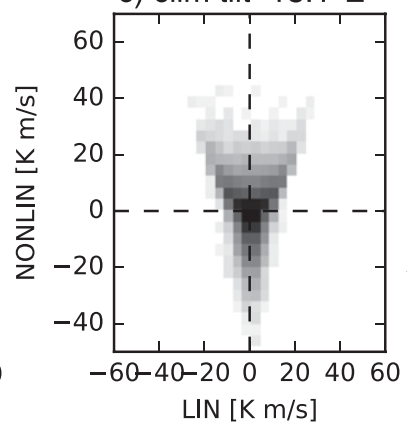

d) $\operatorname{clim}$ tilt $=0^{\circ} \mathrm{E}$

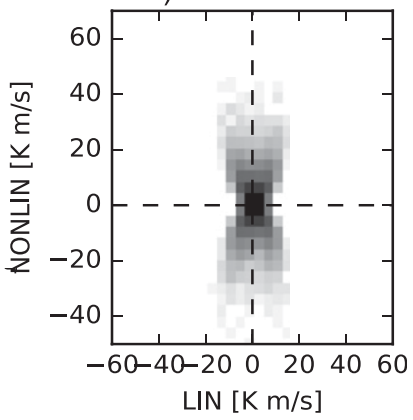

FIG. 8. Two-dimensional histograms of LIN vs NONLIN as computed with the toy model with a climatological tilt of (a) $54.3^{\circ}$, (b) $36.2^{\circ}$ (the observed wave- 1 tilt at $60^{\circ} \mathrm{N}$ and $100 \mathrm{hPa}$ for DJF), (c) $18.1^{\circ}$, and (d) $0^{\circ} \mathrm{E}$. Anomaly parameter distributions are constructed as for toy model 2 (see Table 2).

between the two hemispheres is due to the differences in climatological wave tilt. The Southern Hemisphere's heat flux distribution will be further discussed in the next section.

To confirm that the westward tilt of the climatological wave is responsible for the observed relationship between LIN and NONLIN, Fig. 8 plots 2D histograms between the two terms for four versions of the toy model that are identical except for the climatological wave tilts that are prescribed. The second panel (Fig. 8b) is the same as toy model 2 described above, and it prescribes the observed climatological tilt of $36.2^{\circ}$ (i.e., Fig. $8 \mathrm{~b}$ is just reproducing Fig. $6 \mathrm{a}$ ). The other versions increase (Fig. 8a) or decrease (Figs. 8c and $8 \mathrm{~d}$ ) the climatological wave tilt. Figure 8 shows that, qualitatively, the toy model with the observed climatological tilt has the LIN/NONLIN distribution that looks most like the observed relationship. Furthermore, it confirms that when the climatological wave is equivalently barotropic (i.e., $d \theta_{c} / d \ln p=0$ ), there is no well-defined relationship between the LIN and NONLIN terms (except that when the NONLIN term is close to zero, the LIN term tends to be close to zero as well). The nonmonotonic dependence of skew on the climatological wave tilt (recall Fig. 7) can be understood as follows: for very small climatological tilts, there is no well-defined relationship between the LIN and NONLIN terms, and since for this version of the toy model neither of them has a skew, the total heat flux also has near-zero skew. For more moderate tilts like the observed, the strong relationship between the LIN and NONLIN terms leads to a significant positive skew of the heat flux anomaly. Finally, for even larger tilts (Fig. 8a), the LIN term begins to dominate the total heat flux (i.e., its variance is significantly larger than the NONLIN term's variance) and hence the skew of the total heat flux is closer to the skew of the LIN term, which is zero for this version of the toy model.

\section{f. Additional results}

\section{1) WAVE-2 HEAT FLUX DISTRIBUTION}

The observed wave-2 DJF $\left\{v^{*} T^{*}\right\}^{\prime}$ distribution at $60^{\circ} \mathrm{N}$ and $100 \mathrm{hPa}$ has a similar variance to the wave- 1 distribution (cf. Figs. 1c and 1d) and a somewhat larger positive skewness. Differently than for wave 1, the wave2 LIN distribution has a skew that is not significantly different from zero, while the NONLIN wave-2 skew of 1.61 is similar to the wave-1 NONLIN skew (see Table 1). In this section, we briefly analyze the wave- 2 heat flux distributions.

It was argued in section $3 \mathrm{~d}$ that the cause of the negative skewness of the wave-1 LIN term was the tendency for anomalies out of phase with the climatological wave to be of larger amplitude than in-phase anomalies. Since the observed wave-2 LIN distribution has a skew of nearly zero, this is a useful test case for that argument. Figure 9 shows the average amplitude of observed wave anomalies as a function of their phase, for both wave 1 and wave 2 . As was hinted at by Fig. 4d, wave-1 anomalies that are out of phase with the climatological wave tend to be of slightly higher amplitude than those that are in phase with the climatological wave (Fig. 9a). On the other hand, wave- 2 anomaly amplitudes do not have a clear systematic dependence on phase (Fig. 9b). Given that the wave-1 LIN term has a negative skew but the wave-2 LIN term does not, this supports the argument that the skewness of the LIN term is determined by the relationship between wave anomaly amplitude and phase. To quantify this, the average amplitude of wave anomalies in phase with the climatological wave, specifically those anomalies with 

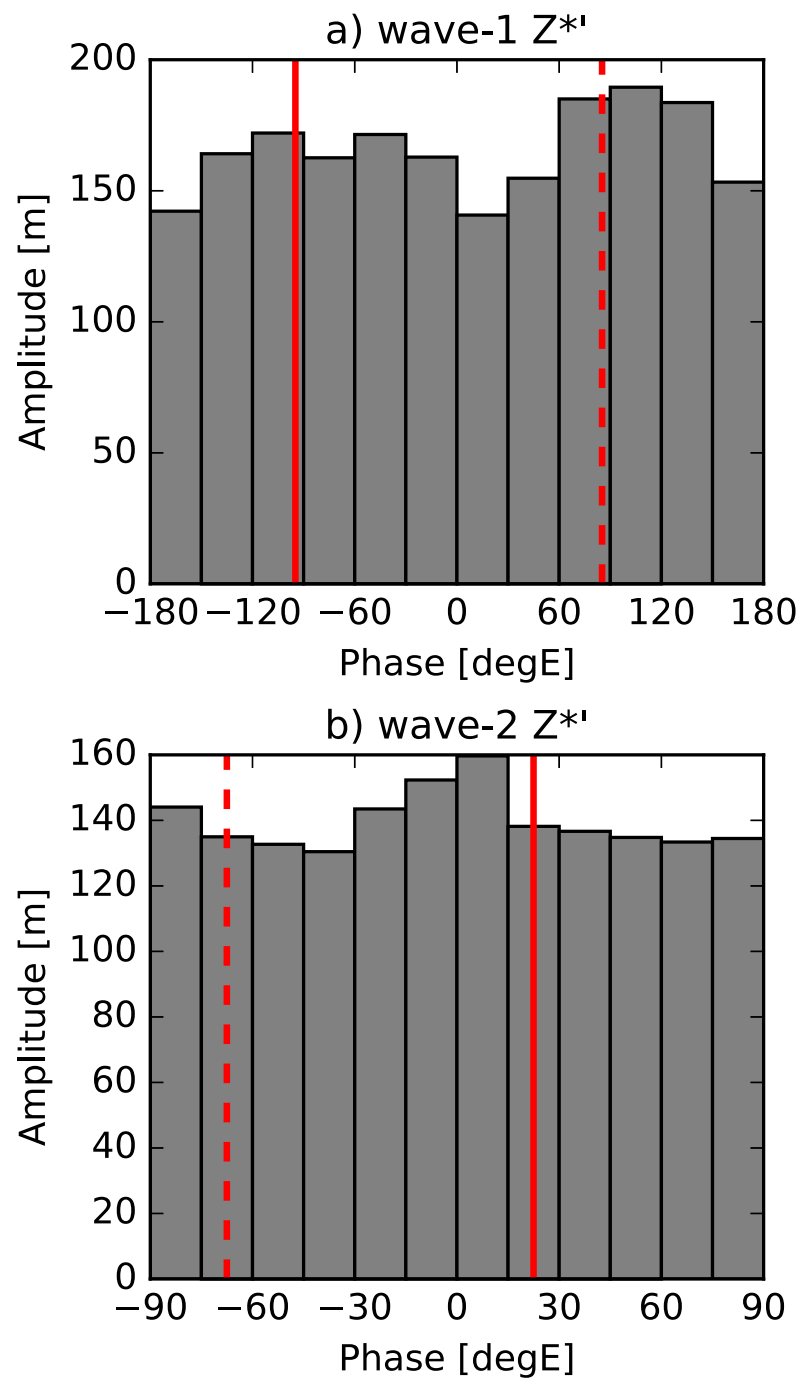

FIG. 9. Average anomaly amplitude when the anomaly phase is within a given bin. Computed for (a) wave- $1 Z^{*^{\prime}}$ and (b) wave- $2 Z^{* \prime}$ at $60^{\circ} \mathrm{N}$ and $100 \mathrm{hPa}$, for all DJF days.

$\left|\phi^{\prime}-\phi_{c}\right|<\pi / 4$, is compared to anomalies out of phase with the climatological wave [i.e., $\left|\phi^{\prime}-\left(\phi_{c}+\pi\right)\right|<\pi / 4$ ]. For wave 1 , in-phase anomalies had an average amplitude of $167 \mathrm{~m}$ and out-of-phase anomalies averaged $188 \mathrm{~m}$. This difference was significant $(p<0.01)$ using Welch's $t$ test, which does not assume equal variances in each sample. On the other hand, for wave 2 in-phase anomalies averaged $137 \mathrm{~m}$ and out-of-phase anomalies averaged $132 \mathrm{~m}$, and this difference was not significant $(p=0.37)$.

\section{2) SOUTHERN HEMISPHERE}

The Southern Hemisphere's polar vortex is known to be substantially less variable than the Northern Hemisphere's polar vortex (e.g., Yoden et al. 2002) and only one sudden stratospheric warming has been observed to occur in the Southern Hemisphere (Newman and Nash 2005). Nevertheless, there is still a substantial amount of upward wave activity flux variability in the Southern Hemisphere, of which the majority is attributable to the LIN term during SON (Fig. 9 of Smith and Kushner 2012). Furthermore, the climatological wave 1 in the Southern Hemisphere's lower stratosphere actually has a larger amplitude than the corresponding Northern Hemisphere component: its amplitude is $203 \mathrm{~m}$ at $60^{\circ} \mathrm{S}$ and $100 \mathrm{hPa}$ averaged over SON, compared to $133 \mathrm{~m}$ at $60^{\circ} \mathrm{N}$ and $100 \mathrm{hPa}$ averaged over DJF. The distribution of wave- 1 heat flux at $60^{\circ} \mathrm{S}$ and $100 \mathrm{hPa}$ over all SON days has a skew of $0.79 \pm 0.11$ (Table 1 ). Overall, the wave-1 heat flux distribution in the Southern Hemisphere is similar to the Northern Hemisphere's distribution, the most significant difference being that the NONLIN part has a skew of only $0.84 \pm 0.22$ compared to $1.76 \pm 0.46$ in the Northern Hemisphere.

The wave-2 heat flux distribution in the Southern Hemisphere also has a positive skew (with a value of $0.75 \pm 0.16$ ), but it is for a different reason than the cases discussed up until now. This is because the wave- 2 climatology in the Southern Hemisphere has an amplitude of only $25 \mathrm{~m}$, compared to $157 \mathrm{~m}$ in the Northern Hemisphere, and is nearly barotropic in the lower stratosphere. Because of the climatology's small amplitude, the NONLIN term explains nearly all of the variance of the wave- 2 heat flux in the Southern Hemisphere (the correlation between the two is $r=0.96$ ). Thus, the wave- 2 heat flux distribution is essentially the same as the wave-2 NONLIN distribution, and the positive skewness can be attributed directly to the skewness of the NONLIN distribution, which is present because of the tendency of westward-tilted wave anomalies to be of larger amplitude (see discussion in section 3d). This is distinct from the Northern Hemisphere wave-1 and wave-2 distributions and the Southern Hemisphere wave- 1 distributions, where the westward tilt of the climatological wave is the most important contribution to the positive skewness of the total heat flux distribution.

\section{Summary and discussion}

This study investigated why the upward wave activity flux distribution in the wintertime polar stratosphere is positively skewed in order to help elucidate how dynamics might control the positive skewness of wintertime temperatures in this region. The motivation for doing so was to understand the distribution of temperature in the stratosphere, which is essential for ozone chemistry and is also related to the extreme changes in stratospheric polar vortex strength, which tend to be 
followed by long-lasting northern annular mode anomalies in the troposphere. The typical explanation for the positive skewness of temperature is that there is a lower bound on temperatures set by a radiative limit, while dynamical wave driving can force large positive anomalies of temperature. In this work it was shown that the heat flux distributions themselves are positively skewed, and it was suggested that this can at least partially explain the positive skewness of temperatures. The primary focus was on the wave- 1 heat flux at $60^{\circ} \mathrm{N}$ and $100 \mathrm{hPa}$, during boreal winter.

The ideas of linear interference were used to understand the heat flux distributions. It was shown that when the heat fluxes are filtered by wavenumber, the LIN and NONLIN terms have a well-defined relationship that can be understood as follows: because the climatological wave has a westward tilt with height, the largest positive and negative LIN days will occur when the anomalous wave also has a westward tilt with height and is either in or out of phase with the climatological wave. This means that the NONLIN term tends to be large and positive when the LIN term is either negative or positive. Thus, when the LIN term is negative (positive), it tends to cancel (amplify) the NONLIN term, and this leads to the positive skewness of the total heat flux anomaly.

To confirm that linear interference plays a role in determining the heat flux skew, a simple toy model was constructed that computes the heat flux distribution given prescribed distributions for the wave anomaly amplitudes, tilts, and phases, as well as values for the climatological wave amplitude, tilt, and phase. Using this model it was shown that 1 ) the skew of the LIN term is due to out-of-phase wave anomalies tending to be of larger amplitude, 2) the skew of the NONLIN term is due to the largest amplitude anomalies tending to be westward tilted, and 3) one can obtain a positively skewed total heat flux distribution without having a skewed LIN or NONLIN distribution, just as a result of the above-described relationship between the two terms. Furthermore, using the toy model with a large range of prescribed climatological wave tilts showed that the heat flux skew has a strong dependence on the climatological tilt and that it goes to zero when the climatological wave becomes barotropic. This suggests that the tilt of the climatological wave could be used as a proxy for the heat flux distribution skew in climate model analysis.

As a final comment, it is known that there is a strong correlation between time-integrated heat flux at $100 \mathrm{hPa}$ and temperature or polar vortex strength (i.e., the NAM) in the midstratosphere (Newman et al. 2001; Polvani and Waugh 2004). However, the connection between daily heat flux and temperatures/NAM is much weaker (Fig. 3 of Polvani and Waugh 2004). Thus, a question may be raised as to the importance of the skewness of the daily heat flux distribution for the skewness of the temperature distribution in the stratosphere. To address this, the skewness of the distribution of the time-integrated heat flux was computed for multiple integration lengths. It is found that although there is a slight dependence of skew on integration length, the skew is always positive. Recall that for daily all-wavenumber $\left\{v^{*} T^{*}\right\}^{\prime}$ at $60^{\circ} \mathrm{N}$ and $100 \mathrm{hPa}$ for all DJF days, the skew was 0.45 (Fig. 1b). For 10-, 20-, and 40-day integrated heat fluxes at the same location, the skews are $0.66,0.73$, and 0.33 , respectively. For wave-1 heat fluxes, the skews for these same integration periods $(10,20$, and 40 days) are $0.80,0.67$, and 0.46 , respectively. Future work will aim to understand in more detail the dependence of the heat flux distribution skewness on the length of time integration, and furthermore the connection between the upward wave activity flux distribution in the lower stratosphere and the temperature (or polar vortex strength) distribution in the midstratosphere.

Acknowledgments. The authors thank Dr. Edwin P. Gerber and three anonymous reviewers for their detailed comments on the manuscript. $\mathrm{O}$. W. is grateful for the discussions with Dr. Frédéric Laliberté, which led to this work. The authors acknowledge the support of the Natural Sciences and Engineering Research Council of Canada and the Ontario Graduate Scholarship program. O. W. was supported by the NOAA Climate and Global Change Postdoctoral Fellowship Program, administered by UCAR's Cooperative Programs for the Advancement of Earth System Science.

\section{REFERENCES}

Andrews, D. G., J. R. Holton, and C. B. Leovy, 1987: Middle Atmosphere Dynamics. International Geophysics Series, Vol. 40, Academic Press, 489 pp.

Baldwin, M. P., and T. J. Dunkerton, 2001: Stratospheric harbingers of anomalous weather regimes. Science, 294, 581-584, https://doi.org/10.1126/science.1063315.

Dee, D. P., and Coauthors, 2011: The ERA-Interim reanalysis: Configuration and performance of the data assimilation system. Quart. J. Roy. Meteor. Soc., 137, 553-597, https://doi.org/ 10.1002/qj.828.

Dunn-Sigouin, E., and T. A. Shaw, 2015: Comparing and contrasting extreme stratospheric events, including their coupling to the tropospheric circulation. J. Geophys. Res. Atmos., 120, 1374-1390, https://doi.org/10.1002/2014JD022116.

Garfinkel, C., D. Hartmann, and F. Sassi, 2010: Tropospheric precursors of anomalous Northern Hemisphere stratospheric polar vortices. J. Climate, 23, 3282-3299, https://doi.org/ 10.1175/2010JCLI3010.1 
Gillett, N. P., M. P. Baldwin, and M. R. Allen, 2001: Evidence for nonlinearity in observed stratospheric circulation changes. J. Geophys. Res., 106, 7891-7901, https://doi.org/10.1029/ 2000JD900720.

Kalnay, E., and Coauthors, 1996: The NCEP/NCAR 40-Year Reanalysis Project. Bull. Amer. Meteor. Soc., 77, 437-471, https:// doi.org/10.1175/1520-0477(1996)077<0437:TNYRP>2.0.CO;2.

Labitzke, K., 1982: On the interannual variability of the middle stratosphere during the northern winters. J. Meteor. Soc. Japan, 60, 124-139, https://doi.org/10.2151/jmsj1965. 60.1_124.

Manney, G. L., and Coauthors, 2011: Unprecedented Arctic ozone loss in 2011. Nature, 478, 469-475, https://doi.org/10.1038/ nature10556.

Newman, P. A., and E. R. Nash, 2005: The unusual Southern Hemisphere stratosphere winter of 2002. J. Atmos. Sci., 62, 614-628, https://doi.org/10.1175/JAS-3323.1.

- — - and J. E. Rosenfield, 2001: What controls the temperature of the Arctic stratosphere during the spring? J. Geophys. Res., 106, 19 999-20 010, https://doi.org/10.1029/2000JD000061.

Nishii, K., H. Nakamura, and T. Miyasaka, 2009: Modulations in the planetary wave field induced by upward-propagating Rossby wave packets prior to stratospheric sudden warming events: A case-study. Quart. J. Roy. Meteor. Soc., 135, 39-52, https://doi.org/10.1002/qj.359.

Polvani, L. M., and D. W. Waugh, 2004: Upward wave activity flux as a precursor to extreme stratospheric events and subsequent anomalous surface weather regimes. J. Climate, 17, 3548-3554, https://doi.org/10.1175/1520-0442(2004)017<3548: UWAFAA $>2.0 . \mathrm{CO} ; 2$.
Shaw, T. A., and J. Perlwitz, 2013: The life cycle of Northern Hemisphere downward wave coupling between the stratosphere and troposphere. J. Climate, 26, 1745-1763, https://doi.org/ 10.1175/JCLI-D-12-00251.1.

_ and - 2014: On the control of the residual circulation and stratospheric temperatures in the Arctic by planetary wave coupling. J. Atmos. Sci., 71, 195-206, https://doi.org/10.1175/ JAS-D-13-0138.1.

Smith, K. L., and P. J. Kushner, 2012: Linear interference and the initiation of extratropical stratosphere-troposphere interactions. J. Geophys Res., 117, D13107, https://doi.org/10.1029/ 2012jd017587

Thompson, D. W. J., and J. M. Wallace, 2000: Annular modes in the extratropical circulation. Part I: Month-to-month variability. J. Climate, 13, 1000-1016, https://doi.org/10.1175/ 1520-0442(2000)013<1000:AMITEC>2.0.CO;2.

von Storch, H., and F. W. Zwiers, 1999: Statistical Analysis in Climate Research. Cambridge University Press, 496 pp.

Watt-Meyer, O., and P. J. Kushner, 2015a: Decomposition of atmospheric disturbances into standing and traveling components, with application to Northern Hemisphere planetary waves and stratosphere-troposphere coupling. J. Atmos. Sci., 72, 787-802, https://doi.org/10.1175/JAS-D-14-0214.1.

- , and $-2015 \mathrm{~b}$ : The role of standing waves in driving persistent anomalies of upward wave activity flux. J. Climate, $\mathbf{2 8}$, 9941-9954, https://doi.org/10.1175/JCLI-D-15-0317.1.

Yoden, S., M. Taguchi, and Y. Naito, 2002: Numerical studies on time variations of the troposphere-stratosphere coupled system. J. Meteor. Soc. Japan, 80, 811-830, https://doi.org/ $10.2151 / \mathrm{jmsj} .80 .811$. 